\title{
EL LENGUAJE DEL CENTRO EDUCATIVO, ELEMENTO IMPULSOR DE LA IGUALDAD DE OPORTUNIDADES ENTRE GÉNEROS: LA FORMACIÓN PERMANENTE DE LA COMUNIDAD EDUCATIVA.
}

\author{
Amando López Valero \\ Eduardo Encabo Fernández \\ Universidad de Murcia
}

RESUMEN. La historia de nuestras sociedades ha conllevado una concepción de las mismas de corte patriarcal. El modelo androcéntrico ha imperado y ello ha provocado en muchos aspectos no sólo la discriminación de la mujer sino también del varón. Desde este artículo, defendemos la teoría de que uno de los elementos que ha contribuido a ese sexismo que ha impregnado la sociedad ha sido el lenguaje. Éste en su relación indisociable con la mente, motiva que con un mal uso del mismo se conformen y perpetúen los estereotipos sexistas. Por todo ello, proponemos el trabajo lingüístico con la Comunidad Educativa en su conjunto para que las nuevas generaciones no se vean salpicadas por este fenómeno social, el cual no debería haber aparecido nunca, ya que, se convierte en elemento legitimador de status y de poder de unos pocos, en detrimento de otras muchas.

Palabras Clave: Lenguaje, Discriminación, Estereotipos, Igualdad de Oportunidades, Género.

ABSTRACT. Our societies history, has put up with a conception of them as patriarchal societies. The adrogynous model has reigned and that has caused in an important number of aspects not only women discrimination but men too. From this article, we defend the theory that one of the elements that has contributed to that discrimination, that has impregnated society has been the language. This, in his indissolubility with mind, motivates that with a bad use of it, it's been shaped and perpetuated discriminating stereotypes. We propose working with the Educative Community for the new generations don't be splashed by this social phenomenon, that never should have appeared because it becomes a legitimating status and power element.

Key Words: Language, Discrimination, Estereotypes, Equality of Opportunities, Gender.

"La lengua es totalmente original y todo el que pone los pies en el terreno de la lengua puede decirse que se ve abandonado por todas las analogías del cielo y de la tierra» (ENGLER, 1968) 
Analizar la Lengua es una cuestión apasionante y supone el verse abandonado a la suerte de estudiar un terreno totalmente abierto a cualquier posibilidad, ya que, en su condición de compañera de viaje del ser humano a lo largo de la vida, pasa por los mismos estadios que este último y tal vez es esa la característica que la hace tan atractiva e interesante en cualquiera de los campos de investigación que nos proporciona. En este trabajo nos adentraremos en la relación que mantiene el lenguaje con el sexismo, veremos cómo ambos se hallan interconexionados y uno influye sobre el otro. La temática referente a la igualdad de oportunidades entre los sexos está recorriendo un camino lento pero seguro hacia su consecución. Tradicionalmente, el prístino rol de la mujer se había limitado a ser de índole abnegado, ahora se está reconociendo que no es un mero objeto decorativo que se halla al servicio del varón, sino que puede alcanzar cotas de desarrollo personal similares al primero, y es más, puede aportar al desarrollo de la sociedad nuevas ideas que hagan prosperar a la misma. Todo esto es una realidad, pero no parece ser suficiente, ya que, la discriminación por razón de género sigue estando presente en nuestra sociedad, y lo hace adoptando distintas formas, entre ellas la ideología, las normas sexuales y el lenguaje. En el uso de este último elemento creemos reconocer a un agente, que no permite la óptima consecución de la igualdad de oportunidades. La adquisición de un lenguaje que no discrimine, que sea más humano, es uno de los retos que se nos plantean a la hora de planificar la educación de las nuevas generaciones. Con el convencimiento de que con tal transición se logrará el objetivo, creemos en la necesidad de formar a la comunidad educativa -como uno de los núcleos de formación de las personas- para que su utilización del lenguaje sea acorde con la defensa de la igualdad de oportunidades, la cual viene prescrita por la L.O.G.S.E. (1990). Procedamos al análisis de la cuestión, reflexionando en primer lugar sobre la importancia que posee el lenguaje en nuestras vidas.

\section{El lenguaje, ese báculo tan necesario en nuestras vidas}

Desde que vemos la luz en este mundo, sentimos la necesidad de comunicarnos con los demás. A través de diferentes modalidades del lenguaje (corporal, hablado,...) nos ponemos en contacto con nuestros semejantes. El lenguaje es pues, una facultad humana que nos permite avanzar en nuestro desarrollo como personas. Acotando el término SAPIR (1986:14) nos dice que el lenguaje es «un método exclusivamente humano, y no instintivo, de comunicar ideas, emociones y deseos por medio de un sistema de símbolos producidos de manera deliberada. Estos símbolos son ante todo auditivos, y son producidos por los llamados "órganos del habla" », la función del lenguaje será completamente instrumental. Es preciso distinguir en el lenguaje entre lengua y habla, la primera se refiere a la forma particular que adquiere el lenguaje en una comunidad social determinada, y el habla es el comportamiento del individuo que pone en práctica su lengua (BRONCKART, 1980). Esta distinción desarrollada por Ferdinand de SAUSSURE va a convertirse en trascendental a la hora de concebir la lingüística tal y como lo hacemos en estos momentos. Con un sencillo símil podríamos explicar de un modo más preciso tal separata, imaginemos una hoja de papel, el pensamiento (lengua) corresponderá al anverso y el sonido (habla) será el reverso. La lengua como concreción del lenguaje, va a cumplir tres funciones básicas: la comu- 
nicativa, la representativa y la reguladora. Las tres funciones se dan de manera interrelacionada y simultánea (REYZÁBAL, 1993). El grado de importancia que alcanza la Lengua no es mensurable, no podemos imaginar la importancia que adquiere, tal vez sea este el motivo -nuestra semi-ignorancia- por el cual no le concedamos la importancia que merece, sólo lo hacemos cuando nos ha resultado imprescindible para la consecución de algún determinado fin. El lenguaje es por tanto, un hecho social y como tal poseerá una funcionalidad, su aprendizaje debe ser consecuente y estar al servicio de la vida de todas las personas (BALLY, 1977). Además, como elemento en constante cambio, no es nada fácil fijar un determinado estado del mismo, describirlo y hacer una enseñanza del mismo sin hacer referencia a los cambios a los que está sujeto (MALMBERG, 1971). Precisamente, en las mutaciones del mismo nos basamos para anticipar un cambio de usos para el Tercer Milenio. No es descabellado concebir una evolución del lenguaje hacia una vertiente más humana, que incluya a todas las personas. Queda pues constatada la relevancia que posee el lenguaje en nuestros modelos vitales de funcionamiento y la no soslayabilidad vital del mismo (LÓPEZ VALERO y ENCABO FERNÁNDEZ, 1999), pasemos a ver ahora cómo esa trascendencia del lenguaje se traduce en una indisociabilidad con el pensamiento humano.

\section{Una relación indisociable: lenguaje-pensamiento}

Mucho se ha debatido sobre la cuestión, ¿el lenguaje condiciona nuestro pensamiento?, o ¿es el pensamiento el que condiciona nuestro lenguaje?. Entablar un debate para dilucidar cuál de las dos posturas es la que posee más argumentos a favor, sería algo dificultoso y que tan sólo nos conduciría hacia la divagación. En este escrito nos posicionaremos en consonancia con la primera de las posturas pues estamos convencidos de que de alguna manera el lenguaje condiciona nuestro pensamiento. Al respecto, la hipótesis de WHORF nos dice que todos los niveles altos de pensamiento dependen del lenguaje, y que la estructura del lenguaje que la persona utiliza habitualmente influye sobre la manera en que ella misma comprende el medio ambiente que le rodea (WHORF, 1971). Con tales premisas podemos observar que el progreso personal irá estrechamente unido a sus avances lingüísticos, y aunque sea un tanto pretencioso el así afirmarlo, su status social dependerá del grado de desarrollo que hayan alcanzado sus competencias tanto lingüística como comunicativa. Profundizando un poco más en la hipótesis Whorfiana, debemos completar la misma con la aportación de Edward SAPIR, dicha fusión nos otorgará la conocida como hipótesis SAPIR-WHORF. Tal propuesta está basada en dos principios: el determinismo lingüístico y la relatividad lingüística. El principio del determinismo lingüístico establece que la lengua tiene la capacidad de determinar el pensamiento, lo cual viene condicionado por la relatividad lingüística: el mundo ofrece un conjunto complicado de imágenes que la mente de las personas percibe y organiza a través de un patrón igual para toda una comunidad de hablantes y que está codificado en las estructuras de su lengua (MARCELLESI y GARDIN, 1979). Convencidos pues de que nuestro pensamiento está ampliamente influenciado por el lenguaje que utilizamos, procedamos a dar aplicación a esta asunción y relacionémosla con la conformación de estereotipos en las personas. 


\section{Realmente, ¿a qué llamamos estereotipo?}

«Conjunto de creencias compartidas sobre las características personales, generalmente rasgos de personalidad, pero también los comportamientos propios de un grupo de personas», así nos definen YZERBYT y SCHADRON (1996) el estereotipo. En cuanto a los estereotipos de género se definen como un conjunto estructurado de pensamientos acerca de los atributos personales de mujeres y varones o de sujetos clasificados como masculinos o femeninos (ASHMORE y DEL BOCA, 1979). La evolución de este tipo de estereotipos va desde la consideración de los atributos masculinos y femeninos como polaridades antagónicas y excluyentes entre sí, hasta la búsqueda de explicaciones alternativas a la falta de comprobación empírica de diferencias comportamentales entre mujeres y varones (BARBERÁ, 1995). Las personas tendemos a funcionar a base de imágenes mentales, de generalizaciones abusivas, de antojos mentales. Es por tal razón que estos estereotipos influyen tanto en la manera en que asimilamos las informaciones nuevas como en el modo en que las recordamos. Podemos afirmar que el estereotipo tiene un importante impacto en la interpretación, adquisición y restitución de las informaciones. La interpretación es partícipe de que las informaciones que nos Ilegan refuercen los estereotipos. Estos pueden perpetuarse igualmente porque proporcionan las premisas sobre las que basamos nuestro comportamiento. Usualmente, los estereotipos hacen formulaciones de cómo son los grupos: los rasgos de personalidad que se cree que comparten sus miembros y las emociones y sentimientos que los miembros del grupo despiertan en los demás (SMITH y MACKIE, 1997). Podemos decir que el estereotipo es un retrato de la mente, una imagen mental simplificada de cómo es visto un determinado grupo y lo que hace. Entre los diversos estereotipos que nuestro pensamiento puede generar, existen los acertados y los desacertados, entre estos últimos hallamos los estereotipos sexistas a los que ya hemos hecho mención, aquellos que nos definen a la mujer (porque del hombre casi nunca han sido estereotipos negativos) como sumisa del hombre, asumiendo unos roles determinados. ¿Cuáles eran estos roles tradicionales?. Los roles femeninos siempre se han relacionado con: gracia-belleza, entrega-abnegación, educación de los hijos, fragilidad-dulzura. En este caso, la preceptora historia ha motivado que haya habido una evaluación negativa del grupo social que constituyen las mujeres. Este grupo a veces ha sido tan maltratado que se lo ha tenido proscrito de la sociedad (QUIN y MCMAHON, 1997). Nuestras creencias sobre lo masculino y lo femenino responden a construcciones sociales. El contenido de los estereotipos de género tiene su origen en las "observaciones" que hace la gente de las actividades realizadas por hombres y mujeres, actividades determinadas en primer lugar por los roles sociales asignados a ella (ECHEBARRÍA y PINEDO, 1997). Entre los diversos elementos que conforman los estereotipos sexistas, consideramos que el lenguaje tiene su parte de responsabilidad, ahora bien, veamos, en qué medida el lenguaje se halla implicado en dicha conformación.

\section{El lenguaje, ¿parte implicada en la conformación de estereotipos sexistas?}

El epígrafe que encabeza este apartado plantea la dicotomía entre la inocencia o la complicidad de la lengua en la conformación de estereotipos sexistas. Nuestra respuesta es clara. Con anterioridad, LÓPEZ VALERO (1995)/ENCABO FERNÁNDEZ (1999), 
hemos considerado al lenguaje como el factor determinante de la conformación de los estereotipos sexistas. Si bien es cierto que anatómica y fisiológicamente la diferencia de sexos es un dato natural (HÉRITIER, 1996), ello no debe implicar un trato distinto en función del mismo. Históricamente, ha existido un género dominante -el masculino- el cual ha poseído los mayores privilegios. Esa posición privilegiada ha sido facilitada en gran medida por el uso de un lenguaje masculino genérico omnicomprensivo. El avance conjunto indisociable de lenguaje y sociedad, motiva que el lenguaje refleje cómo es la sociedad que lo usa. Los rasgos sexistas de una lengua son fiel espejo de la sociedad. Si bien, como hemos expresado antes, la diferencia sexual está ya dada en el mundo, no es el lenguaje quien la crea, éste se sitúa como un vertebrador de ideas, en este caso, de estereotipos sexistas (LÓPEZ VALERO, 1995), pero lo hace de un modo dirigido por el sentir general de la sociedad que lo utiliza. El entramado es complejo ya que abarca más aspectos que tan sólo el lenguaje y el sexismo. Como nos indican LÓPEZ VALERO y MADRID IZQUIERDO (1998), tales categorías conceptuales se unen a la ideología y a la educación en un andamiaje que subrepticia la discriminación en razón de género. Así pues, el uso sexista de la Lengua, transmitido en todos los ambientes en los que se desenvuelve la persona, incluido el educativo, nos va a conducir a una discriminación no deseada tanto de la mujer como del hombre. Un mal uso del lenguaje motiva que éste se convierta en cómplice del proceso de discriminación social en razón del sexo. La impregnación de sexismo se halla en muchos de los recovecos de la sociedad, evidentemente el grado es cambiante en cada Cultura, se puede apreciar en países del Sur de América donde el sexismo es algo que se hace más acusado que en la zona europea (MORENO OLIVOS y ENCABO FERNÁNDEZ, 1998), pero es algo contra lo que se está alzando la voz y debemos poner los medios para erradicar tal situación de desigualdad. Uno de los primeros medios es claro, el cambio del uso del lenguaje, nuestra ambición debe ser el generar un uso lingüístico que represente a ambos géneros, que nombre las experiencias en un lenguaje sensato, un lenguaje que no subordine, que no infravalore, que no excluya, que no quite la palabra a nadie,..., (VV, AA., 1995), en definitiva, un lenguaje más humano que configure un pensamiento abierto y acorde con los marcos legislativos que rigen nuestra sociedad. La orientación patriarcal debe ser modificada, pero no hacia un modelo matriarcal sino a un modelo igualitario. Las asunciones que la sociedad hace considerando al hombre como combativo, agresivo, lúcido, creador, decidido, emprendedor, tenaz firme, apto para la ciencia, amante del riesgo y la lucha, con capacidad de organización, poseedor de cualidades como actividad, seguridad en sí mismo, autonomía, estabilidad, control de las emociones, ... y a la mujer como frívola, pasiva, dependiente, afectiva, inestable emocionalmente, sensible, abnegada, sumisa, intuitiva, tierna, poseedora de un yo débil y construido en función de los demás, deseosa de agradar a los demás, y en ella priman los aspectos afectivos sobre los intelectuales,... GARCÍA MESEGUER (1994) señaló cómo -a su juicio- la cultura patriarcal inculca muy pronto a las niñas y a los niños la falsa idea de que los géneros gramaticales se corresponden con los sexos, con lo que se otorga ventaja al hombre sobre la mujer a través del papel asimétrico que los dos géneros desarrollan. Como educadores, mostraremos desde este texto la incidencia escolar del sexismo en el lenguaje. Creemos que el centro escolar puede ser un elemento de cambio, y como tal la Comunidad Escolar que le rodea agente de transformación social. Es preciso que 
las nuevas generaciones hereden una educación bañada por el lenguaje apuntado anteriormente: el lenguaje humanizante. Pero claro, previamente a plantear cómo sería la formación permanente de la Comunidad Educativa, es pertinente conocer el estado de la cuestión, ¿cuál es el pensamiento actual de los componentes que inciden en el centro educativo?. Tenemos que averiguar tal sentir para planificar cuál será el trabajo a desarrollar.

\section{5. ¿Cuál es la actitud de la Comunidad Educativa ante estos planteamientos?}

Todavía las personas no somos conscientes de los efectos discriminadores que puede tener un lenguaje genérico masculino. Es por ello que al preguntar a la comunidad educativa sobre la utilización del mismo, la mayoría de las personas hacen alusión al mismo como algo nimio, una moda pasajera, no le confieren la trascendencia que realmente alcanza. No podemos pedir a la Comunidad que inmediatamente entienda la situación, pero hay algo que es evidente, el estrecho vínculo que une lenguaje y sociedad, relaciona de un modo indefectible lo lingüístico y lo psicosocial y podemos comprobar cómo en la Lengua todo es psicológico, en palabras de SAUSSURE (1973): la lingüística forma parte de la psicología social. Es por esa razón que siempre debemos considerar las acciones que pueda acometer el lenguaje en los procesos de relación humana. Como antes hemos dicho, la escuela reproduce consciente e inconscientemente el modelo sexista de la sociedad en la que se encuentra, el profesorado de la misma no admite que su discurso se halla impregnado de sexismo, bajo el pretexto de que esta denuncia es un artilugio del movimiento feminista, se refugian y continúan de ese modo con su instrucción del alumnado de un modo significativamente sexista. Pero no solamente es el profesorado el que lleva a cabo, esta educación que únicamente legitima y perpetúa el modelo patriarcal sino que de igual modo la familia colabora en seguir formando a las nuevas generaciones bajo el prisma de lo masculino. El objetivo sigue siendo el matenimiento del modelo androcéntrico. Realmente, no debería ser así, legislativamente se reconoce la igualdad de género, pero como estamos defendiendo durante todo este texto, la relación bidireccional pensamiento-lenguaje no permite que esa igualdad pueda ser un hecho hasta que el uso del lenguaje no conlleve la misma. Es competencia del Consejo Escolar el idear un Proyecto Educativo de Centro donde se priorice la detección del sexismo en el lenguaje y se elimine el mismo. Una buena planificación en ese sentido, repercutirá en la puesta en escena de los contenidos que el Proyecto Curricular describa. No es fácil modificar la mentalidad de los centros educativos, en un reciente estudio LÓPEZ VALERO y MADRID IZQUIERDO (1998) analizaron cuál era el discurso del profesorado ante la transmisión de estereotipos sexistas, y los resultados derivaron en contestaciones por parte del mismo acerca de que el tema está superado y el sexismo se halla menos presente en la escuela. No creemos que esto sea cierto, tal vez esa negación de una realidad responda al temor al cambio. Es preciso hacer la denuncia de estos sucesos porque si no no es posible efectuar la transición, se impone la formación de profesorado, padres y madres, representantes de la administración,..., para que, de igual modo que a ellos de un modo sibilino e inconsciente les ha sido transmitido un modelo androcéntrico, ellos puedan transmitir también de un modo inconsciente un modelo igualitario que no discrimine en razón de sexo. Ahora bien, ¿es suficiente sólo con 
la formación de estos componentes de la Comunidad Educativa?. Evidentemente hay que formarlos desde una perspectiva crítica, para que sepan discernir entre lo que puede perjudicar a sus hijos e hijas y lo que no (LÓPEZ VALERO, MADRID IZQUIERDO y ENCABO FERNÁNDEZ, 1998a), pero de igual modo alcanzan gran trascendencia los materiales curriculares que se puedan utilizar en el centro, las infraestructuras, espacios y tiempos del currículum, el nunca nombrado, pero siempre presente currículum oculto, los prejuicios del profesorado a la hora de la evaluación de sus alumnos y alumnas. Todos ellos componentes de la Cultura particular del centro, la cual se mantiene a la par de la actuación de las personas y la cual es tan importante como la actuación de las primeras. Veamos a continuación cuáles son los elementos del currículum y del Centro Escolar en general que se hallan impregnados por el sexismo.

\section{6. ¿Qué elementos del centro escolar se hallan impregnados de un lenguaje sexista?}

Lógicamente, si hablamos de elementos marcados por la presencia del sexismo, debemos de hablar en primer lugar del pensamiento del profesorado, de su discurso. Es un hecho, el trasfondo de las respuestas del mismo ante preguntas referidas a la presencia del sexismo en las aulas denota que es más cuestión estética que real el contestar que no existe sexismo en las aulas, y más extensamente en la sociedad. Comenzando por Educación Infantil, pasando por Educación Primaria, llegando a Educación Secundaria, y al ámbito universitario, nuestro sistema educativo está cargado de manifestaciones sexistas (LÓPEZ VALERO, MADRID IZQUIERDO y ENCABO FERNÁNDEZ, 1998b). Otro aspecto del currículum -entendido éste en su acepción más extensa- lo constituye la utilización de materiales escolares, concretamente libros de texto, donde la presencia del sexismo se hace bastante acusada. Sin tener que ser reconocidos exegetas, podemos comprobar que los libros de texto usados por el alumnado y profesorado comportan un lenguaje sexista que conduce a la discriminación (LÓPEZ VALERO, 1992/C.O.E.N.S., 1992). Pero dentro de ellos, no sólo es el lenguaje escrito el que rezuma sexismo sino que el lenguaje iconográfico, con la presencia de menos imágenes de género femenino con relación al masculino es otra de las señas de identidad de estos libros utilizados. Más factores que se aúnan en pos de un clima patriarcal en el Centro Educativo, son en el currículum explícito la adecuación de unas asignaturas más al perfil masculino -véase asignaturas de corte técnicoy otras al femenino en función de una clasificación estereotípica basada en la legitimación de poder por parte del modelo androcéntrico. Los espacios del recinto escolar también adquieren su importancia en lo que concierne a la discriminación en razón de sexo, la repartición de los mismos es bastante diferente, tanto es así que existen lugares que prácticamente adquieren el calificativo de tabú para el género femenino como pueda ser por ejemplo el campo de fútbol, además la distribución del patio nunca suele ser igualitaria. Existen dentro del denominado currículum oculto, diferencias de trato a la hora de dirigirse a un género o al otro, así, un ejemplo lo constituye la entonación distinta que el profesorado hace para dirigirse a unos y otras, además puede apreciarse que el lenguaje usado para con las niñas es más adjetivado. Las manifestaciones de sexismo en la escuela son innumerables, sería tarea harto prolija el enumerarlas todas, pero creemos que sabiendo que uno de los factores que más conlleva sexismo es el lenguaje, y trabajando de un modo adecuado con el mismo, es 
posible lograr la ansiada transición. No es cuestión onerosa el tratar de cambiar una situación actual que se viene repitiendo desde hace muchos siglos atrás, se trata de un acto volitivo, de humildad, y de cambiar la concepción del mundo, no centrando todos los anhelos en el poder y el dominio sobre el género contrario. Vistas las condiciones previas genéricas -aunque luego habrá que contextualizar cada centro, el cual se constituirá en un caso particular- de partida, hay que empezar a marcar las directrices de trabajo con la Comunidad Educativa para la extinción de ese, tan nombrado en este texto, sexismo, por ello debemos presentar algunas actuaciones concretas y la metodología general de trabajo para con la Comunidad.

\section{7. ¿Cómo trabajar con la Comunidad de modo que la situación pueda cambiar?}

No es sencillo idear una forma de intervención que sea la panacea y sirva como "recetario" para la eliminación del sexismo. Como nos apuntan BONAL y TOMÉ (1996), el alcance y la efectividad de la coeducación dependerá de dos factores fundamentales: la posición política respecto a la igualdad entre los sexos y la lógica del diseño del proceso de cambio. Respecto al primer aspecto, si entramos a valorar aspectos legislativos, es prescriptivo que pongamos los medios para el logro de la igualdad de oportunidades entre ambos géneros y respecto al segundo de los puntos, debemos secuenciar de una manera lógica nuestra intervención con toda la Comunidad Educativa. Obviamente, no vamos a lograr un proceso catártico con nuestra actuación, pero, poco a poco, podemos ir derruyendo los cimientos de la sociedad patriarcal para transformarla en la sociedad de todas y todos o todos y todas -es indiferente-. El lenguaje, en su innatismo de instrumento de intercambio social, debe mostrar su lado más positivo y el correcto uso del mismo no va a conducir a la pretendida igualdad entre géneros. Nuestro proceso habrá de ser paulatino y seguro. Siguiendo el modelo que nos plantea BONAL (1997), estableceremos dos fases en nuestra actuación, una primera fase de sensibilización -dependerá del grado de sexismo existente en el centro- donde analizaremos las manifestaciones de sexismo que se hacen presentes en la escuela, y una segunda donde trabajaremos con la conocida metodología surgida a finales de los ochenta y principio de los noventa, denominada Investigación-Acción. Coincidimos con GONZÁLEZ HERNÁNDEZ (1989) en que tal metodología es la más adecuada para el trabajo en Ciencias Sociales, y evidentemente la educación se halla inserta en dicho campo de actuación. En la primera fase, la referida a la sensibilización, trataremos de hallar en el centro manifestaciones sexistas, comenzando por el lenguaje público que se utiliza en el mismo, hay que concienciar a todos los miembros del mismo que la disposición semántica y pragmática del lenguaje que se realiza comporta discriminación. Podemos comenzar por observar cómo la presencia de los términos referidos al género femenino es ínfima, cómo es el genérico masculino el que ocupa casi todos los documentos y enunciaciones. Este trabajo es factible de ser llevado a cabo con todos y todas, sería por ello conveniente la creación de una escuela de padres y madres para el trabajo con los mismos y la habilitación de unos espacios y unos tiempos para el trabajo con el profesorado. Un segundo aspecto a trabajar con la Comunidad Educativa sería el de analizar los libros de texto y materiales didácticos. Se ha demostrado que la presencia del sexismo se hace presente en los mismos, pero es mejor que padres y madres, profesorado 
y administración comprueben que existen de un modo muy acentuado denotaciones estereotípicas en la gran mayoría de los materiales que se usan en el centro educativo. Un tercer rasgo que sería interesante de analizar es la distribución y uso de los espacios, los implicados y las implicadas en esta observación podrán comprobar cómo existe una relación de poder de los niños sobre las niñas en un espacio libre. La tarea no será muy complicada ni laboriosa, bastará con confeccionar una lista de control o escala de estimación para comprobar los sucesos sexistas en relación con la utilización de los espacios. Podemos también destacar de entre las múltiples alternativas que tenemos para sensibilizar a la Comunidad, la visión de las producciones que pueda hacer el alumnado, en ellas se apreciará claramente lo influenciado que por la sociedad patriarcal éste se halla. Las pruebas de libre asociación de campos semánticos pueden otorgar una visión más esclarecedora sobre los estereotipos conformados respecto a hombres y mujeres que tiene el alumnado del centro (LÓPEZ VALERO y MADRID IZQUIERDO, 1998). De igual modo, es factible ver cómo las conductas agresivas -por asignación social- corresponden al género masculino y las afectivas al género femenino. Con el recorrido de éstos y otros aspectos, trataremos de sensibilizar a la Comunidad Educativa, con el fin de prepararla para el posterior trabajo en pos de la erradicación del apuntado sexismo presente en la escuela. ¿Cómo será el posterior trabajo?. Como hemos reseñado, la Investigación-Acción debe guiar nuestros procesos de desarraigamiento, ésta supone «una forma de indagación autorreflexiva de los participantes (maestros, estudiantes o directores, por ejemplo) en situaciones sociales (incluyendo las educativas) para mejorar la racionalidad y justicia de: a) sus propias prácticas sociales o educativas, b) la comprensión de tales prácticas, y c) las situaciones (en instituciones) en que estas prácticas se realizan (aulas o escuelas por ejemplo)» (KEMMIS y MCTAGGART, 1988:1). El modelo de proceso seguido en la misma comenzará con una definición del problema y una construcción de hipótesis, cosa que la Comunidad escolar ya habrá efectuado al sensibilizarse con la problemática, por tanto deberá planificar las acciones a desarrollar para solucionar tal problema. Posteriormente interpretará los resultados de esa actuación, los observará y analizará. Reflexionará y evaluará la situación, para volver a subsanar las cosas que no hayan quedado solucionadas. Esta práctica de la Investigación se convierte en un proceso cíclico compuesto por dos fases: la constructiva y la reconstructiva, una basada en el discurso y la otra en la práctica. En un tema en el que tratamos con el pensamiento de las personas, precisaremos de una metodología flexible que nos permita afrontar cualquier imprevisto o situación inesperada que nos pueda surgir. Una vez delimitada, la metodología a seguir, propongamos algunas sugerencias para su aplicación en la práctica diaria de los Centros Educativos. Con el fin de empezar a colocar las bases de un lenguaje más humano, que comprenda a ambos géneros, podemos utilizar términos epicenos como alumnado, profesorado,..., podremos utilizar abstractos como secretaría o asesoría. No es muy acertada, pero otra de las opciones es la de utilizar la siempre farragosa duplicación (los/as). Respecto al trato con el alumnado, es recomendable hablarle de vosotros y vosotras. En relación a otros de los puntos, los materiales curriculares, LÓPEZ VALERO (1992), propone la cuidadosa elección de materiales curriculares a utilizar en el aula, ya sean vídeos didácticos, libros de texto,..., de tal forma que en ellos la mujer y el hombre aparezcan un número similar de ocasiones. Haciendo un poco más de hincapié en las propuestas escolares, MAÑERU 
MÉNDEZ y RUBIO HERRÁEZ (1992) indican que es posible trabajar con la familia, labor que deberá ser facilitada por las reuniones periódicas con la misma, y de igual modo con la creación de la apuntada anteriormente Escuela de padres y madres; las dramatizaciones o las críticas sobre anuncios televisivos o películas son otras de las actividades que nos sugieren dichas autoras. Como vemos, es extensa la relación de mejoras que se pueden efectuar para paliar los efectos del sexismo en nuestras escuelas, la cuestión es, que requerimos de actos volitivos que nos acerquen hacia espacios comunes de actuación que fomenten la solidaridad y por ende la igualdad entre los sexos.

\section{Perspectivas futuras.}

Una doble cara contempla -a nuestro juicio- la situación futura con relación a la temática. Por una parte, es positivo el que se alcen voces de denuncia contra el sexismo en nuestra sociedad comenzando por el ámbito escolar, pero por otro lado, la situación sigue siendo preocupante, ya que, la sociedad patriarcal se sigue imponiendo. La presencia de un lenguaje ya asentado, conformador de estereotipos sexistas, motiva que sea complicado el eliminar dichos modelos de funcionamiento grupal. Porque a lo mejor lo que observamos en el primer caso -el de las voces alzadas- es la cima de un gran iceberg, con un extenso número de mujeres que callan y otorgan una superioridad al hombre, que además éste ha sabido conservar gracias al tejido de araña que con las construcciones sociales se ha edificado. Lo cierto es que el papel de la mujer en las sociedades ha mejorado, pero no tanto como para pensar que el problema de la discriminación está solventado. Decía ROUSSEAU (1978) que lo que el ser humano pierde por el contrato social es su libertad natural y un derecho voluntario a todo lo que le tienta y está a su alcance; y lo que gana es la libertad social y la propiedad de todo lo que posee. Pues bien, esa máxima no se cumple en el caso del género femenino, ya que, la opresión a la que ha sido sometida ha provocado que no tuviese libertad social alguna. El reto está presente, hacer de esta sociedad, un ente más justo e igualitario, y el comienzo lo tenemos claro: cambiar nuestro lenguaje, para que de ese modo se modifique nuestro pensamiento. Posteriormente, podremos entrar en el cambio de otros estamentos para que el sexismo deje de ser una realidad en nuestra sociedad.

\section{Referencias Bibliográficas}

ASHMORE, R. D. y DEL BOCA, F. K. (1979): "Sex stereotypes and implicit personality theory: toward a cognitive-social psychological conceptualization". Sex roles, 5, pp. 219-248.

BALLY, C. (1977): El lenguaje y la vida. Buenos Aires: Losada.

BARBERÁ, E. (1991): "Motivación e identidad de género". En CHÓLIZ, M. Y BARBERÁ, E. (Eds.): Prácticas de psicología de la motivación y de la emoción. Valencia: Promolibro.

B.O.E. (1990): Ley Orgánica 1/1990, de 3 de Octubre, de Ordenación General del Sistema Educativo (B.O.E. 4 de Octubre de 1990). Madrid. 
BONAL, X. y TOMÉ, A. (1996): "Metodologías y recursos de intervención". Cuadernos de Pedagogía, 245, pp. 56-69.

BONAL, X. (1997): Las actitudes del profesorado ante la coeducación. Propuestas de intervención. Barcelona: Graó.

BRONCKART, J. P. (1980): Teorías del lenguaje. Barcelona: Herder.

C.O.E.N.S. (1992): El sexismo iconográfico en los libros de texto. Murcia: Colectivo Escuela no Sexista de Murcia.

ECHEBARRÍA, A. y PINEDO, J. A. (1997): "Identidad social de género: su distribución social e influencia en el juicio". Revista de Psicología Social, (12) 2, pp. 131-151.

ENCABO FERNÁNDEZ, E. (1999): "El lenguaje, elemento configurador de estereotipos sexistas: ¿una cuestión retórica o una diáfana realidad? ". Interlingüística, 10.

ENGLER, R. (1968): Édition critique du cours de linguistique générale de Ferdinand de Saussure. Wiesdoben: Otto Hanasowitz.

GARCÍA MESEGUER, A. (1994): ¿Es sexista la lengua española?. Una investigación sobre el género gramatical. Barcelona: Paidós.

GONZÁLEZ HERNÁNDEZ, A. (1989): La Investigación- Acción como metodología de las Ciencias Sociales. Murcia: Cossío.

HÉRITIER, F. (1996): Masculino/Femenino. El pensamiento de la diferencia. Barcelona: Ariel.

KEMMIS, S. y MCTAGGART, R. (1988): ¿Cómo planificar la Investigación Acción? Barcelona: Laerta.

LÓPEZ VALERO, A. (1992): Lenguaje y discriminación sexista en los libros escolares. Murcia: Universidad de Murcia.

- (1995): "El lenguaje y su influencia en la configuración de los estereotipos sexistas en las personas". En GUERRERO RUIZ, P. y LÓPEZ VALERO, A.: Aspectos de Didáctica de la Lengua y la Literatura. Murcia: Universidad de Murcia.

LÓPEZ VALERO, A. y ENCABO FERNÁNDEZ, E. (1999): "El taller de Lengua y Literatura como medio didáctico para el logro de los objetivos establecidos por el marco legislativo de la L.O.G.S.E. en Educación Primaria". En LÓPEZ VALERO, A., MEROÑO ESPINOSA, C. y PASTOR NOGUERA, M.: Experiencias en la didáctica de la Lengua Castellana y la Literatura. Murcia: CPR Cartagena-La unión.

LÓPEZ VALERO, A. y MADRID IZQUIERDO, J. M. (1998): Lenguaje, sexismo, ideología y educación. Murcia: KR.

LÓPEZ VALERO, A., MADRID IZQUIERDO, J. M. y ENCABO FERNÁNDEZ, E. (1998a): "La formación del profesorado de Lengua y Literatura, desde una perspectiva crítica, para la no transmisión de estereotipos sexistas". En Actas del congreso Igualdad Lingüística: el sexismo en el lenguaje. Málaga. (En prensa).

(1998b): "El sexismo en la enseñanza de la Lengua española y la Literatura (Educación Infantil, Educación Primaria, Educación Secundaria Obligatoria y 
Bachillerato). En Actas del congreso lgualdad Lingüística: el sexismo en el lenguaje. Málaga. (En prensa).

MALMBERG, B. (1971): Los nuevos caminos de la lingüística. Madrid: Siglo XXI.

MAÑERU MÉNDEZ, A. y RUBIO HERRÁEZ, E. (1992): Educación para la igualdad de oportunidades entre ambos sexos. Madrid: M.E.C.

MARCELLESI, J.B. y GARDIN, B. (1979): Introducción a la sociolingüística. La lingüística social. Madrid: Gredos.

MORENO OLIVOS, T. y ENCABO FERNÁNDEZ, E. (1998): “¿Se halla impregnado de sexismo el lenguaje que conforma las Leyes Federales de educación en Argentina y México?". En Actas del congreso Igualdad Lingüística: el sexismo en el lenguaje. Málaga. (En prensa).

QUIN, R. y MCMAHON, B. (1997): Historias y estereotipos. Madrid: Ediciones de la Torre.

REYZÁBAL, M. V. (1993): "La lengua, eje de formación individual y social". Bordón, 45 (1), pp. 59-68.

ROUSSEAU, J. J. (1978): El contrato social. Madrid: Aguilar.

SAPIR, E. (1986): El lenguaje. Introducción al estudio del habla. Madrid: Ediciones F.C.E.

SAUSSURE, F. (1973): Curso de lingüística general. Buenos Aires: Losada.

SMITH, E. R. y MACKIE, D. M. (1997): Psicología social. Madrid: Panamericana.

VV.AA. (1995): En femenino y en masculino. Murcia: Ministerio de Trabajo y Asuntos Sociales. Instituto de la Mujer.

WHORF, B. L. (1971): Lenguaje, pensamiento y realidad. Barcelona: Barral.

YZERBYT, V. y SCHADRON, G. (1996): "Estereotipos y juicio social". En BOURHIS, R. Y. y LEYENS, J. P.: Estereotipos, discriminación y relaciones entre grupos. Madrid: McGraw-Hill. 